\title{
Negative reinforcement via motivational withdrawal is the driving force behind the transition to addiction
}

\author{
Olivier George • George F. Koob • \\ Leandro F. Vendruscolo
}

Received: 18 December 2013 / Accepted: 1 February 2014 / Published online: 13 June 2014

(C) Springer-Verlag Berlin Heidelberg 2014

Piazza and Deroche-Gamonet have significantly contributed to the field of addiction and published several pioneering articles that have had a major influence on the field. The latest article by Piazza and Deroche-Gamonet is a position paper, in which they argue that they provide a foundation for the first general theory of the transition to addiction. Their theory is composed of the following three principles: (1) The transition to addiction depends on an interaction between individual vulnerability and drug exposure. (2) The transition to addiction involves at least three steps (i.e., recreational/sporadic drug use, intensified/sustained/escalated drug use, and loss of control/full addiction). (3) Addiction is a true psychiatric disease. Piazza and Deroche-Gamonet propose to test their "first" general theory of addiction by providing three predictions that can be used to validate or invalidate their theory. The review by Piazza and Deroche-Gamonet represents an excellent opportunity to discuss critical aspects of the transition to addiction. In this article, we attempt to test point by point the validity of their statements based on the current state of the field, with the hope that a better understanding of the addiction process will lead to better treatments for drug addiction.

To test the validity of their claims, as suggested by Piazza and Deroche-Gamonet, it is important to define the criteria that need to be fulfilled for a novel theory to be valid. A scientific theory is a well-substantiated explanation of a phenomenon based on knowledge that has been repeatedly confirmed through observation and experimentation. Its strength

O. George $(\bowtie) \cdot$ G. F. Koob $\cdot$ L. F. Vendruscolo

Committee on the Neurobiology of Addictive Disorders, Scripps

Research Institute, 10550 North Torrey Pines Road, SP30-2400, La

Jolla, CA 92037, USA

e-mail: ogeorge@scripps.edu

Present Address:

G. F. Koob

National Institute on Alcohol Abuse and Alcoholism, Rockville, MD, USA is related to the diversity of phenomena it can explain and the accuracy in predicting outcomes. A scientific theory should allow for falsifiable predictions. Finally, a new theory should better explain experimental observations than previous theories and result in further testable predictions that can be confirmed. We will review each principle proposed by Piazza and Deroche-Gamonet based on each of these criteria.

\section{Principles}

First principle

"The transition to addiction results from an interaction between individual vulnerability and the degree/amount of exposure." Although we understand that the topic of individual vulnerability vs. the degree/amount of drug exposure might have been a matter of debate a few decades ago, this relationship has been extensively tested and investigated to the point that it now is safe to say that this is actually not a theory anymore but a law of behavioral neuroscience. Extensive research has repeatedly demonstrated that behavioral phenotypes, including drug-related behaviors, are modulated by the interaction between individual vulnerability (whether genetic or not) and the environment, including drug availability/ exposure (e.g., Crabbe et al. 1999; Hughes et al. 2011; Redolat et al. 2009; Verweij et al. 2010; Spanagel 2009; Nielsen and Kreek 2012; Agrawal and Lynskey 2008; Ellenbroek et al. 2005; Caprioli et al. 2007; Nader and Czoty 2005; Ahmed 2005; Volkow and Li 2005).

Second principle

"The transition to addiction is composed of at least three steps: recreational sporadic (ReS), intensified, sustained, escalated (ISuE), and loss of control (LoC)." Although the acronyms 
used by Piazza and Deroche-Gamonet are novel, the concept behind them is not. Numerous authors have theorized that the transition to addiction involves the same three steps of use (i.e., the initial first step of reinforcement that is recreational and social and involves learning the reinforcing effects of the drug), abuse (i.e., the second step of increased seeking, consolidation of learning, and escalation of drug intake), and dependence (i.e., the third step with maintenance of escalated intake with loss of control, full addiction, or Dependence). Numerous epidemiological studies have used these three steps of use, abuse, and dependence to characterize the transition to addiction (for details, see Chapter 1, "Definitions of Addictions: Drug Use, Drug Abuse, and Drug Addiction," of Koob and Le Moal 2006). Additionally, below is a non-exhaustive list of authors who have not only included these three steps using different terms but also provided additional steps to better explain the addiction process, including withdrawal and relapse that are essential to the transition to addiction.

- Kreek et al. (2002): (1) initial use of drug, (2) sporadic intermittent use, (3) regular use, (4) addiction, (5) early withdrawal/relapse, (6) protracted abstinence/relapse/ sustained abstinence.

- Koob and Le Moal (2006): (1) acute reinforcement/social drug taking, (2) escalating/compulsive use, (3) dependence, 4) withdrawal, (5) relapse/recovery.

- Everitt et al. (2001): (1) vulnerability; (2) the maintenance of drug-taking/seeking behavior, which might be viewed as a dynamic product of the gradual strengthening or "consolidation" of behavior that arises from the reinforcing action of drugs, supplemented by a recurrent shaping of drug-related memories; (3) the eventual progression of addiction to a form of habit-based learning through which voluntary control over drug use is lost; (4) the propensity for relapse of drug seeking/taking, which often occurs after protracted abstinence.

- Kalivas and O'Brien (2008): (1) social use, (2) regulated relapse, (3) compulsive relapse.

Thus, a more reasonable argument is that the general theory proposed by Deroche-Gamonet embraces a concept that has indeed (a) been with us for a long time and (b) evolved substantially. Indeed, the recent elimination of the "abuse" designation in DSM-V is a reflection of the evolution of the hypothesis that addiction represents a continuum of excessive drugtaking, from recreational use to severe Substance Use Disorder.

\section{Third principle}

"The transition to addiction is a true psychiatric disease." The overwhelming majority of neuroscientists would agree that addiction is a true psychiatric disorder. The debate between the iatrogenic and psychiatric views of addiction as outlined by Piazza and Deroche-Gamonet has added value, however, in elegantly applying multiple arguments with which to persuade those few academic holdouts and the general public.

In summary, our only objection to the general theory that addiction is a psychiatric disorder that results from an interaction between the individual and the environment that progresses along at least three steps of use, abuse, and addiction with loss of a control over drug intake, is that it is not particularly novel, has already been proposed in numerous articles, and been accepted by the most prominent scientists in the field. Therefore, it is difficult to see how it can better explain experimental observations than previous theories. Piazza and Deroche-Gamonet's general theory provides a well-substantiated explanation of the importance of individual differences, and this specific hypothesis has been repeatedly confirmed through observation and experimentation. The notion of "protection" or what we might call "resilience" is definitely an area for future research.

\section{Predictions}

Despite the fact that the three principles proposed by Piazza and Deroche-Gamonet are already well-known facts that are accepted by the overwhelming majority of scientists, let us assume that these principles are novel and test the validity of the three predictions as suggested by Piazza and DerocheGamonet. As mentioned by the authors, for a theory to be valid it must provide falsifiable predictions; however, when a falsifiable prediction is not possible to be proven wrong by definition, such as "no human lives forever," or is only falsifiable in theory but not in practice, such as "it will be raining here in one billion years," it invalidates the falsifiable prediction and makes a theory unscientific (Popper 2005).

\section{First prediction}

"The transition to addiction depends on an interaction between individual vulnerability and drug exposure." The authors specifically state, "variation in the degree of these interactions cannot be seen as a fundamental fallacy of the theory," and that one must prove "that one of these two variables is not necessary in the development of pathological drug use." If variation in the degree of interaction is not a fallacy, then for this prediction to be falsifiable one must demonstrate that drug addiction can occur in an individual without access to the drug or that drug addiction can occur without an individual. By definition, drug addiction requires an individual who has or had access to a drug; therefore, this prediction is unfalsifiable.

\section{Second prediction}

"The transition to addiction is a process that develops along at least three steps (recreational, intensified, loss of control)." A 
transition is the process of changing from one state to another. There is a minimum of two steps that describe a transition, the original state and the final state. Therefore, for this prediction to be falsifiable, one would have to demonstrate that the transition from drug use to drug addiction is instantaneous without a transition state. While this prediction is falsifiable in theory, it is not falsifiable in practice because chemical and biological phenomena involved in neuronal activity exhibit transitional states. From a simple chemical reaction, such as the change from adenosine triphosphate to adenosine diphosphate, to more complex phenomena, such as the activation of neurotransmitter receptors, the generation of action potentials, the release of neurotransmitters, or neuronal remodeling, all of these phenomena exhibit transitional states.

\section{Third prediction}

"The transition to addiction is a true psychiatric disease." The authors add that to falsify their theory, one must "demonstrate that in most conditions drug exposure is both necessary and sufficient to induce addiction." "Most conditions" is a vague term that cannot be tested experimentally; therefore, this prediction is not falsifiable.

As mentioned by the authors, for a theory to be valid, it must provide falsifiable predictions, unfortunately the predictions provided by Piazza and Deroche-Gamonet do not appear to be falsifiable. However, the fact that the predictions provided by Piazza and Deroche-Gamonet are not falsifiable does not mean that they are not relevant. We agree with the statements that addiction results from the interaction between the individual and the drug, that it develops along at least three steps, and that it is a true psychiatric disease, but these are not falsifiable predictions, and we encourage the authors to elaborate more specific predictions that can be tested experimentally.

\section{Where we disagree ... somewhat}

\section{Embracing hedonic allostasis but discarding "withdrawal"}

Piazza and Deroche-Gamonet state, "we have not proposed as relevant models of transition to addiction important drug-induced physiological adaptations, such as tolerance or withdrawal, which are diagnostic criteria for SUD but are not necessary or sufficient conditions for a diagnosis of the disease." While we agree that these adaptations are not necessary or sufficient for the diagnosis of Addiction, it is important to realize that the diagnostic criteria in the DSM-IV or DSM-V are not relevant to the understanding of the mechanisms that underlie the progression of a disease. One can imagine that the best clinical symptom(s) to identify an individual with a specific disease may be a mere consequence of reaching the ultimate stage of the disease and unrelated to the cause of the transition to the ultimate stage of the disease. Clinicians know that the earlier a disease is treated, the better the outcome. By focusing on only a sufficient criterion (loss of control) and excluding neuroadaptations and behaviors that are critical for the pathological process (tolerance, withdrawal, and bingeing), one risks missing the unique neuroadaptation that is actually causing the transition to addiction before the clinical symptoms have fully developed and that could be the target of novel treatments, particularly for individuals who have a history of abuse, but fail to meet the diagnostic criteria for severe substance-use disorder. The National Institute of Mental Health (NIMH) already recognized the fallacy of excluding biological adaptations based on DSM criteria by no longer funding research projects that rely exclusively on DSM criteria, and we strongly encourage researchers to investigate phenomena that precede the loss of control over drug intake. Moreover, tolerance and negative emotional states during withdrawal are particularly important phenomena that deserve special attention. Even if they are not sufficient or necessary criteria to characterize addiction, they are phenomena that represent a powerful driving force to increase the motivational properties of drugs of abuse. While we agree with the authors that physical withdrawal is not necessarily observed in all drug addicts, it is important to understand that withdrawal is a multifaceted phenomenon that includes both physical and emotional symptoms and that drugs of abuse that were considered to be associated with very little if any physical withdrawal, such as cocaine or marijuana, are now known to be associated with a very strong emotional withdrawal, including anxiety, irritability, and hypohedonia (D'Souza and Markou 2010; Budney et al. 2004) that not only provide a driving force to loss control over drug intake through negative reinforcement (Cohen et al. 2013) but also represent potential vulnerability for individual differences for the transition to addiction (Koob et al. 2013, 2014; George and Koob 2010). Disregarding animal models that emphasize emotional withdrawal would be a critical error that would most likely delay progress on the neurobiological mechanisms that underlie drug addiction and the development of novel therapeutic strategies. Indeed, we would further argue that the state of loss of reward in hedonic allostasis to which Piazza and DerocheGamonet refer and the state of "mourning" to which they refer are indeed simply what we have hypothesized for over 15 years as "motivational withdrawal." While Koob and colleagues have been unable to extricate the colonic 
blockage of "physical withdrawal" from the metabolism of theories of addiction, we keep trying. Indeed, we are heartened to see it replaced by hedonic allostasis and mourning.

\section{What happened to the brain stress systems?}

Piazza and Deroche-Gamonet repeatedly invoke negative reinforcement in the form of self-medication as a mechanism for enjoying the pleasures of drug taking in the human population. However, they fail to bridge this construct to the transition to addiction. For example, they wrote, "One of the most easily identified functions of drugs is their stress relieving and anxiolytic effects, which certainly have an important role in helping individuals function in most human societies that are largely very demanding, often unjust, and practically never egalitarian." While they elegantly and correctly outline the allostatic view of addiction of Koob and Le Moal, they leave out half of the story. We have argued, with some substantial evidence, that as dependence and withdrawal develop, brain anti-reward systems, such as corticotropin-releasing factor (CRF), norepinephrine, and dynorphin, are recruited in the extended amygdala to produce a negative emotional state from the side of stress, malaise, and pain that we believe also accounts for a significant amount of motivational withdrawal or what Piazza and Deroche-Gamonet call "mourning." For example, extracellular CRF in the extended amygdala is increased during acute withdrawal from drugs of abuse. Critically, CRF receptor antagonists injected into the extended amygdala block the anxiety-like effect of drug withdrawal and blunt excessive drug taking during escalated drug taking with extended access (Koob 2003). We have hypothesized for over 15 years and demonstrated that the brain stress neurotransmitter CRF, which is known to be activated during the development of excessive drug taking, comprises a betweensystem opponent process. This activation is manifest when the drug in removed, producing anxiety, hyperkatifeia, and irritability symptoms associated with acute and protracted abstinence (George et al. 2007; Edwards et al. 2012; Cohen et al. 2013). Recent data show that blockade of the $K$ opioid system can also block the aversive stimulus effects of drug withdrawal and stress, and excessive escalated drug self-administration can also be blocked by K antagonists (Koob 2013; Chartoff et al. 2012; Walker et al. 2011; Wee et al. 2009; Schlosburg et al. 2013). These effects may be mediated by the shell of the nucleus accumbens (Nealey et al. 2011; Schlosburg et al. 2013) and central nucleus of the amygdala (Gilpin et al. 2013; Kallupi et al. 2013). These results suggest a within-/ between-system neuroadaptation that was originally hypothesized by Carlezon and Nestler (Carlezon et al. 1998), in which activation of CREB by excessive dopamine and opioid peptide receptor activation in the nucleus accumbens trigger the induction of dynorphin to feedback to suppress dopamine release. Thus, anti-reward circuits are recruited as betweensystem neuroadaptations (Koob and Bloom 1988; George et al. 2012a, b) during the development of addiction, producing aversive or stress-like states (Nestler 2001; Koob 2003; Aston-Jones et al. 1999) via two mechanisms: direct activation of stress-like, fear-like states in the extended amygdala (CRF-norepinephrine) and indirect activation by suppressing dopamine (dynorphin).

\section{Classification of the model of escalation of drug intake as an animal model that precedes the transition to loss of control}

Piazza and Deroche-Gamonet define the animal model of escalation of drug intake after extended access to the drug developed by Ahmed and Koob (1998) as being an animal model of "intensified-sustained-escalated use" that has no relevance as a model of "loss of control of drug use or full addiction." If we follow the authors' own logic, then an animal model of the full addiction stage must meet the criteria for drug addiction developed in the DSM-IV and now in the DSM-V. By analogy, the escalation model in our laboratory and other laboratories has been shown to exhibit seven of the seven items in the DSM-IV and seven of the 11 items in the DSM-V, including most of the criteria required for severe use disorder: (1) tolerance (Ben-Shahar et al. 2005), (2) withdrawal (Ahmed et al. 2002; Vendruscolo et al. 2011), (3) substance taken in larger amount than intended (Ahmed and Koob 1998), (4) unsuccessful efforts to quit (Ahmed and Cador 2006; Lenoir and Ahmed 2007), (5) considerable time spent to obtain the drug (Wee et al. 2008), (6) important social, work or recreational activities given up because of use (George et al. 2008; Vendruscolo et al. 2011; Lenoir et al. 2013), (7) continued use despite adverse consequence (Xue et al. 2012; Vanderschuren and Everitt 2004; Vendruscolo et al. 2011; Vendruscolo et al. 2012; Seif et al. 2013; Lenoir and Ahmed 2007; Ahmed 2012). Clearly, after significant escalation of drug intake, this is an animal model of loss of control over drug intake. Even more than that, we believe that when taken as a whole it is one of the most useful animal models to date to study the transition to addiction as one can investigate the three different steps of use (initial limited access), abuse (escalation of intake), and addiction (escalated intake) in the same paradigm. However, and in contrast to Piazza and Deroche-Gamonet, we do not believe that our animal model is the only relevant model to study the compulsive aspect of drug addiction or is a perfect model of drug addiction in humans. Other groups, including Piazza, Ahmed, Wise, Roberts, and Miczek, to name a few, have generated very interesting animal models that capture different aspects of 
compulsive drug intake (Morgan et al. 2005; Ahmed 2012; Tornatzky and Miczek 2000; Deroche-Gamonet et al. 2004).

We agree with Piazza and Deroche-Gamonet that the transition to addiction requires two types of vulnerability, one for the abuse of drugs and one for the loss of control of intake. We predict that these two types of vulnerabilities can be detected by analyzing the change in cocaine intake in rats during the transition from limited to extended access to drugs. Contrary to Piazza's theory, we predict that the escalation of drug intake in this animal model will be associated with not only a quantitative change but also a qualitative change in drug intake. We also predict that escalation in drug intake is not attributable to an increase in the initial vulnerability to take the drug but rather in the loss of this initial vulnerability and development of a new vulnerability of loss of control over drug intake that follows the switch from positive to negative reinforcement.

\section{A somewhat myopic view of the biological bases of loss of control}

The authors state, "the only biological modification yet specifically associated with loss of control of drug intake is a loss of synaptic plasticity." We have demonstrated above that rats in the escalation model exhibit all of the criteria required for addiction, including loss of control. The authors ignore a vast amount of literature showing neuroadaptations in animals that exhibit the escalation of alcohol, nicotine, cocaine, methamphetamine, and heroin intake. For instance, $100+$ articles from at least 19 different laboratories, including G.F. Koob, T.E. Robinson, J. Mantsch, K.A. Miczek, C.M. Weiss, R.E. See, S.R. Jones, R.M. Carrelli, M. Marinelli, P.J. Kenny, M.T. Bardo, M. Roberto, A. Ettenberg, L.H. Parsons, S. H. Ahmed, B.M. Walker, N.W. Gilpin, C.D. Mandyam, P.V. Piazza, V. Deroche-Gamonet, and O. George, have been published using the escalation model, including biological measures also observed in humans, such as reduced dopamine function (Briand et al. 2008; Schwendt et al. 2009), hypofrontality (George et al. 2008; Briand et al. 2008; George et al. 2012a, b; Meinhardt et al. 2013), and most importantly from our perspective, changes in extrahypothalamic and neuroendocrine stress systems (Adinoff et al. 1990; Vendruscolo et al. 2012). Indeed, the finding that gabapentin, a drug that was first found to be effective in reducing alcohol drinking in rats with escalation of alcohol drinking in a dependence model and had a profile in the central nucleus of the amygdala similar to a $\mathrm{CRF}_{1}$ antagonist (Roberto et al. 2008), was recently found to also be effective in humans with alcohol dependence (Mason et al. 2013) demonstrates the predictive validity of the escalation model for the loss of control over drug intake. Piazza and Deroche-Gamonet missed this literature. While we all focus on our own piece of the addiction cycle, general theories of addiction need to cast a broader net of conceptual frameworks.

\section{Percentage of vulnerable individuals}

The authors state that their animal model of loss of control exhibits the same percentage of vulnerable animals $(\sim 15$ $20 \%$ ) as in humans. This fact is used as a powerful argument for face validity. Moreover, they argue that extending access to the drug (escalation model) does not increase the percentage of rats that show loss of control, making it an argument in favor of their theory that escalation of drug intake is not associated with loss of control. Unfortunately, there are two major problems with their analysis. First, the percentage of animals with loss of control is mathematically predetermined by the fact that they use three behaviors that are highly correlated with each other (typically with $R=0.80-0.90$ ) and that they use a cut-off of the upper $\sim 35 \%$ to diagnose a positive criterion. Therefore, the percentage of animals with loss of control is mathematically restricted to a very narrow range of values. To demonstrate this point, we generated normally distributed random numbers using the Box-Muller transformation (Mayes 2010) and simulated the percentage of animal with loss of control (LoC) depending on the correlation coefficients between the three behaviors using the same threshold ( $35 \%$ ) used by Piazza and Deroche-Gamonet. The results are the following: $R=1.0, \mathrm{LoC}=35 \% ; R=0.97, \mathrm{LoC}=$ $29 \% ; R=0.87 ; \mathrm{LoC}=22 \% ; R=0.35, \mathrm{LoC}=10 \%$. Our simulation found a very similar level of $\mathrm{LoC}$ animals ( $\sim 22 \%)$ when using correlation coefficients similar to Piazza and DerocheGamonet's studies $(R=0.87)$. Combining this simulation with a $\chi^{2}$ power analysis (power $=0.8$, alpha $=0.05$ ), one would require 626 rats per group to obtain a significant difference between $22 \%$ and $29 \%$. Therefore, it is virtually impossible to demonstrate any increase in the percentage of rats with loss of control using their model. The fact that resistance to punishment may follow a bimodal distribution will not change this fact. The reason why Piazza and Deroche-Gamonet found this percentage to be very resistant to changes, including extending access to the drug, is not because the escalation model is not associated with loss of control, and it is not because it is a true measure of who is vulnerable to the loss of control. It is because it is a biased measure that is mathematically constrained to produce a very restricted range of values close to what the authors believe to be the percentage of individuals who are vulnerable to loss of control in humans. The second problem is that we believe that the authors are incorrect when they compare the percentage of users who become addicted in humans and rats while having two completely different environments. If humans were locked inside their homes with legal and free chronic daily access to drugs as the only daily activity, with no access to any alternative reinforcers other than water and bland food, then it would be extremely surprising if the percentage of drug-addicted users would stay at $\sim 20 \%$. Moreover, recent statistics indicate that the percentage of individuals who meet the criteria for Substance Use Disorders of 
those who ever used during the past year ranges from $9.8 \%$ for alcohol to $65.5 \%$ for heroin. The only drug in the $20 \%$ range is cocaine (Koob et al. 2013, 2014).

In conclusion, the theory developed by Piazza and Deroche-Gamonet is not exceedingly novel. Indeed, its major premise is widely accepted in academia in the United States. While we agree with most of their analysis of the literature, we strongly disagree with their view that only animal models that reflect the DSM-IV/V are relevant for the transition to addiction. The key to solve the enigma of drug addiction is not whether we can produce addiction in every single individual, whether there is more than two steps in the transition to addiction, or whether addiction is a psychiatric disorder. All of these questions have already been answered. The keys to solve this enigma are (1) to investigate neuroadaptations associated with different aspects of the transition to addiction, including incentive-salience, tolerance, motivational withdrawal, escalation, cognitive impairment, and loss of control, not only over drug intake but also loss of control over emotion, stress, and pain; (2) to determine the neuronal networks and plasticity (or lack thereof) that mediate the vulnerability to seek and take drugs at every single step of the addiction process as well as relapse after abstinence; (3) to develop novel therapeutic approaches that can reduce compulsive drug seeking and taking in individuals with addiction and return the brain motivational systems to homeostasis; and (4) to use various animal and human models for every stage of the addiction process to identify resistance to the transition to addiction and provide an evidence-based approach to prevention.

\section{References}

Adinoff B, Martin PR, Bone GH, Eckardt MJ, Roehrich L, George DT, Moss HB, Eskay R, Linnoila M, Gold PW (1990) Hypothalamicpituitary-adrenal axis functioning and cerebrospinal fluid corticotropin releasing hormone and corticotropin levels in alcoholics after recent and long-term abstinence. Arch Gen Psychiatry 47:325-330

Agrawal A, Lynskey MT (2008) Are there genetic influences on addiction: evidence from family, adoption and twin studies. Addiction 103:1069-1081

Ahmed SH (2005) Imbalance between drug and non-drug reward availability: a major risk factor for addiction. Eur J Pharmacol 526:9-20

Ahmed SH (2012) The science of making drug-addicted animals. Neuroscience 211:107-125. doi:10.1016/j.neuroscience.2011.08. 014

Ahmed SH, Cador M (2006) Dissociation of psychomotor sensitization from compulsive cocaine consumption. Neuropsychopharmacology 31:563-571

Ahmed SH, Koob GF (1998) Transition from moderate to excessive drug intake: change in hedonic set point. Science 282:298-300
Ahmed SH, Kenny PJ, Koob GF, Markou A (2002) Neurobiological evidence for hedonic allostasis associated with escalating cocaine use. Nat Neurosci 5:625-626

Aston-Jones G, Delfs JM, Druhan J, Zhu Y (1999) The bed nucleus of the stria terminalis. A target site for noradrenergic actions in opiate withdrawal. Ann N Y Acad Sci 877:486-498

Ben-Shahar O, Moscarello JM, Jacob B, Roarty MP, Ettenberg A (2005) Prolonged daily exposure to i.v. cocaine results in tolerance to its stimulant effects. Pharmacol Biochem Behav 82:411-416

Briand LA, Flagel SB, Garcia-Fuster MJ, Watson SJ, Akil H, Sarter M, Robinson TE (2008) Persistent alterations in cognitive function and prefrontal dopamine D2 receptors following extended, but not limited, access to self-administered cocaine. Neuropsychopharmacology 33:2969-2980

Budney AJ, Hughes JR, Moore BA, Vandrey R (2004) Review of the validity and significance of cannabis withdrawal syndrome. Am J Psychiatry 161:1967-1977

Caprioli D, Celentano M, Paolone G, Badiani A (2007) Modeling the role of environment in addiction. Prog Neuropsychopharmacol Biol Psychiatry 31:1639-1653

Carlezon WA Jr, Thome J, Olson VG, Lane-Ladd SB, Brodkin ES, Hiroi N, Duman RS, Neve RL, Nestler EJ (1998) Regulation of cocaine reward by CREB. Science 282:2272-2275

Chartoff E, Sawyer A, Rachlin A, Potter D, Pliakas A, Carlezon WA (2012) Blockade of kappa opioid receptors attenuates the development of depressive-like behaviors induced by cocaine withdrawal in rats. Neuropharmacology 62:167-176

Cohen A, Treweek J, Edwards S, Leão RM, Schulteis G, Koob GF, George O (2013) Extended access to nicotine leads to a CRF(1) receptor dependent increase in anxiety-like behavior and hyperalgesia in rats. Addict Biol

Crabbe JC, Phillips TJ, Buck KJ, Cunningham CL, Belknap JK (1999) Identifying genes for alcohol and drug sensitivity: recent progress and future directions. Trends Neurosci 22:173-179

Deroche-Gamonet V, Belin D, Piazza PV (2004) Evidence for addictionlike behavior in the rat. Science 305:1014-1017

D'Souza MS, Markou A (2010) Neural substrates of psychostimulant withdrawal-induced anhedonia. Curr Top Behav Neurosci 3:119-178

Edwards S, Vendruscolo LF, Schlosburg JE, Misra KK, Wee S, Park PE, Schulteis G, Koob GF (2012) Development of mechanical hypersensitivity in rats during heroin and ethanol dependence: alleviation by $\mathrm{CRF}_{1}$ receptor antagonism. Neuropharmacology 62:1142-1151

Ellenbroek BA, van der Kam EL, van der Elst MC, Cools AR (2005) Individual differences in drug dependence in rats: the role of genetic factors and life events. Eur J Pharmacol 526:251-258

Everitt BJ, Dickinson A, Robbins TW (2001) The neuropsychological basis of addictive behaviour. Brain Res Brain Res Rev 36:129-138

George O, Koob GF (2010) Individual differences in prefrontal cortex function and the transition from drug use to drug dependence. Neurosci Biobehav Rev 35:232-247

George O, Ghozland S, Azar MR, Cottone P, Zorrilla EP, Parsons LH, O'Dell LE, Richardson HN, Koob GF (2007) CRF-CRF 1 system activation mediates withdrawal-induced increases in nicotine selfadministration in nicotine-dependent rats. Proc Natl Acad Sci U S A 104:17198-17203

George O, Mandyam CD, Wee S, Koob GF (2008) Extended access to cocaine self-administration produces long-lasting prefrontal cortex-dependent working memory impairments. Neuropsychopharmacology 33:2474-2482

George O, Le Moal M, Koob GF (2012a) Allostasis and addiction: role of the dopamine and corticotropin-releasing factor systems. Physiol Behav 106:58-64

George O, Sanders C, Freiling J, Grigoryan E, Vu S, Allen CD, Crawford E, Mandyam CD, Koob GF (2012b) Recruitment of medial prefrontal cortex neurons during alcohol withdrawal predicts cognitive 
impairment and excessive alcohol drinking. Proc Natl Acad Sci U S A 109:18156-18161

Gilpin NW, Roberto M, Koob GF, Schweitzer P (2013) Kappa opioid receptor activation decreases inhibitory transmission and antagonizes alcohol effects in rat central amygdala. Neuropharmacology 77C:294-302

Hughes K, Quigg Z, Eckley L, Bellis M, Jones L, Calafat A, Kosir M, van Hasselt N (2011) Environmental factors in drinking venues and alcohol-related harm: the evidence base for European intervention. Addiction 106:37-46

Kalivas PW, O'Brien C (2008) Drug addiction as a pathology of staged neuroplasticity. Neuropsychopharmacology 33:166-180

Kallupi M, Wee S, Edwards S, Whitfield TW Jr, Oleata CS, Luu G, Schmeichel BE, Koob GF, Roberto M (2013) Kappa opioid receptor-mediated dysregulation of gamma-aminobutyric acidergic transmission in the central amygdala in cocaine addiction. Biol Psychiatry 74:520-528

Koob GF (2003) Neuroadaptive mechanisms of addiction: studies on the extended amygdala. Eur Neuropsychopharmacol 13:442-452

Koob GF (2013) Negative reinforcement in drug addiction: the darkness within. Curr Opin Neurobiol 23:559-563

Koob GF, Bloom FE (1988) Cellular and molecular mechanisms of drug dependence. Science 242:715-723

Koob GF, Le Moal M (2006) Neurobiology of addiction. Academic Press, London

Koob GF, Kandel D, Baler RD, Volkow ND (2013) Pathophysiology of addiction. In: Tasman A, Kay J, Lieberman JA, First MB, Maj M (Eds), Psychiatry, 4th edition. Wiley, Chichester, in press

Koob GF, Buck CL, Cohen A, Edwards S, Park PE, Schlosburg JE, Schmeichel B, Vendruscolo LF, Wade CL, Whitfield TW Jr George O (2014) Addiction as a stress surfeit disorder. Neuropharmacology 76:370-382

Kreek MJ, LaForge KS, Butelman E (2002) Pharmacotherapy of addictions. Nat Rev Drug Discov 1:710-726

Lenoir M, Ahmed SH (2007) Heroin-induced reinstatement is specific to compulsive heroin use and dissociable from heroin reward and sensitization. Neuropsychopharmacology 32:616-624

Lenoir M, Cantin L, Vanhille N, Serre F, Ahmed SH (2013) Extended heroin access increases heroin choices over a potent nondrug alternative. Neuropsychopharmacology 38(7):1209-1220. doi:10.1038/ npp.2013.17

Mason BJ, Quello S, Goodell V, Shadan F, Kyle M, Begovic A (2013) Gabapentin treatment for alcohol dependence: a randomized clinical trial. JAMA Intern Med

Mayes (2010)Generating correlated normally distributed random numbers in Excel. JFMET

Meinhardt MW, Hansson AC, Perreau-Lenz S, Bauder-Wenz C, Stählin O, Heilig M, Harper C, Drescher KU, Spanagel R, Sommer WH (2013) Rescue of infralimbic mGluR2 deficit restores control over drug-seeking behavior in alcohol dependence. J Neurosci 33:2794 2806

Morgan D, Smith MA, Roberts DC (2005) Binge self-administration and deprivation produces sensitization to the reinforcing effects of cocaine in rats. Psychopharmacology (Berl) 178(2-3):309316

Nader MA, Czoty PW (2005) PET imaging of dopamine D2 receptors in monkey models of cocaine abuse: genetic predisposition versus environmental modulation. Am J Psychiatry 162:1473-1482

Nealey KA, Smith AW, Davis SM, Smith DG (2011) K-opioid receptors are implicated in the increased potency of intra-accumbens nalmefene in ethanol-dependent rats. Neuropharmacology 61:35-42
Nestler EJ (2001) Molecular basis of long-term plasticity underlying addiction. Nat Rev Neurosci 2:119-128

Nielsen DA, Kreek MJ (2012) Common and specific liability to addiction: approaches to association studies of opioid addiction. Drug Alcohol Depend 123:S33-S41

Popper K (2005) The logic of scientific discovery. Routledge, London

Redolat R, Pérez-Martínez A, Carrasco MC, Mesa P (2009) Individual differences in novelty-seeking and behavioral responses to nicotine: a review of animal studies. Curr Drug Abuse Rev 2:230-242

Roberto M, Gilpin NW, O'Dell LE, Cruz MT, Morse AC, Siggins GR, Koob GF (2008) Cellular and behavioral interactions of gabapentin with alcohol dependence. J Neurosci 28:5762-5771

Schlosburg JE, Whitfield TW Jr, Park PE, Crawford EF, George O, Vendruscolo LF, Koob GF (2013) Long-term antagonism of $k$ opioid receptors prevents escalation of and increased motivation for heroin intake. J Neurosci 33:19384-19392

Schwendt M, Rocha A, See RE, Pacchioni AM, McGinty JF, Kalivas PW (2009) Extended methamphetamine selfadministration in rats results in a selective reduction of dopamine transporter levels in the prefrontal cortex and dorsal striatum not accompanied by marked monoaminergic depletion. J Pharmacol Exp Ther 331:555-562

Seif T, Chang SJ, Simms JA, Gibb SL, Dadgar J, Chen BT, Harvey BK, Ron D, Messing RO, Bonci A, Hopf FW (2013) Cortical activation of accumbens hyperpolarization-active NMDARs mediates aversion-resistant alcohol intake. Nat Neurosci 16:1094-1100

Spanagel R (2009) Alcoholism: a systems approach from molecular physiology to addictive behavior. Physiol Rev 89:649-705

Tornatzky W, Miczek KA (2000) Cocaine self-administration "binges": transition from behavioral and autonomic regulation toward homeostatic dysregulation in rats. Psychopharmacology (Berl) 148(3): 289-298

Vanderschuren LJ, Everitt BJ (2004) Drug seeking becomes compulsive after prolonged cocaine self-administration. Science 305:10171019

Vendruscolo LF, Schlosburg JE, Misra KK, Chen SA, Greenwell TN, Koob GF (2011) Escalation patterns of varying periods of heroin access. Pharmacol Biochem Behav 98:570-574

Vendruscolo LF, Barbier E, Schlosburg JE, Misra KK, Whitfield TW Jr, Logrip ML, Rivier C, Repunte-Canonigo V, Zorrilla EP, Sanna PP, Heilig M, Koob GF (2012) Corticosteroid-dependent plasticity mediates compulsive alcohol drinking in rats. J Neurosci 32:7563-7571

Verweij KJ, Zietsch BP, Lynskey MT, Medland SE, Neale MC, Martin NG, Boomsma DI, Vink JM (2010) Genetic and environmental influences on cannabis use initiation and problematic use: a metaanalysis of twin studies. Addiction 105:417-430

Volkow N, Li TK (2005) The neuroscience of addiction. Nat Neurosci 8: $1429-1430$

Walker BM, Zorrilla EP, Koob GF (2011) Systemic K-opioid receptor antagonism by nor-binaltorphimine reduces dependence-induced excessive alcohol self-administration in rats. Addict Biol 16:116-119

Wee S, Mandyam CD, Lekic DM, Koob GF (2008) Alpha 1-noradrenergic system role in increased motivation for cocaine intake in rats with prolonged access. Eur Neuropsychopharmacol 18:303-311

Wee S, Orio L, Ghirmai S, Cashman JR, Koob GF (2009) Inhibition of kappa opioid receptors attenuated increased cocaine intake in rats with extended access to cocaine. Psychopharmacology (Berl) 205: 565-575

Xue Y, Steketee JD, Sun W (2012) Inactivation of the central nucleus of the amygdala reduces the effect of punishment on cocaine selfadministration in rats. Eur J Neurosci 35:775-783 